\title{
Magnesium sulphate as first line and mainstay therapy without sedation in the management of a patient with tetanus
}

\author{
VE G Dassanayake ${ }^{I^{*}}$ \\ Senior Lecturer in Anaesthesiology, Faculty of Medicine, University of Colombo, Sri Lanka.
}

\section{Corresponding author: viharadassanayake@gmail.com}

\begin{abstract}
We report a patient diagnosed as grade I tetanus that rapidly progressed to grade III and was treated successfully with magnesium sulphate for 19 days without sedation with benzodiazepines.
\end{abstract}

Keywords: Tetanus; magnesium suphate; without sedation; early tracheostomy; successful recovery

\section{Introduction}

Inspite of widespread immunization programs in Sri Lanka, it is still not unusual for tetanus patients to be admitted to District Base hospitals. Due to limited resources, long duration and severity of the disease, and the likely complications, mortality rates remain high. According to statistics from the Epidemiology unit at the Ministry of Health in Sri Lanka there were 15 clinically confirmed cases of tetanus in 2010 with 3 deaths. In 2014 the number had reduced to 10 but with 5 deaths. This justifies the continuing need for researching the management of tetanus with a view to minimizing mortality.

Magnesium has been advocated as the first line therapy to treat both spasms and autonomic dysfunction in severe tetanus. ${ }^{1}$ However, this regime is not yet universally accepted and most authors recommend benzodiazepines and muscle relaxants for the control of spasms. ${ }^{2,3}$ The current WHO recommendation is to use large dose diazepam for control of spasms and magnesium and morphine for the control of sympathetic over action. This combination could be life threatening. ${ }^{4}$ Recent intensive care guidelines highlight the value of a conscious, co-operative patient to avoid the dangers of heavy sedation, and magnesium therapy for tetanus has been highly recommended. ${ }^{5}$

We report the successful management of grade III tetanus with magnesium as mainstay therapy without sedation.

\section{Case presentation}

A 55 year old farmer was transferred to the surgical ward of our District Base hospital from a local hospital with a history of fever on and off for one week associated with severe myalgia. $\mathrm{He}$ had gradually developed difficulty in swallowing and limited mouth opening of 3 days duration. He had no obvious wounds on examination but he claimed to have sustained a thorn prick injury over the sole of the right foot one week back. He had not received immunization against tetanus within the last 10 years.

On examination he was febrile and exhibited neck stiffness, trismus and generalised muscle rigidity. The Spatula Test was positive and confirmed the diagnosis of tetanus. On admission his GCS was 15/15 and bilateral pupils were size 3 equally reactive to light. His blood pressure was $140 / 90 \mathrm{mmHg}$ with a pulse rate of 92 beats per minute, regular rhythm and of good volume. Arterial saturation on air was $97 \%$ and his respiratory rate was 14 breaths per minute with vesicular breathing on auscultation. He had increased muscle tone on all four limbs and exaggerated reflexes with no evidence of sensory loss. According to the Ablett classification of severity of tetanus patient belonged to grade I (mild) disease severity.

Following admission to the surgical ward he was referred to the ICU team and we decided on an early tracheostomy and a bed on ICU was reserved for further care. Our intention was to perform an immediate tracheostomy. Due to logistical reasons in a resource limited setting we had to electively intubate him initially in theatre, as his airway was likely to get compromised. A naso-gastric tube was inserted the same time to enable early feeding. His haemodynamic parameters remained stable during the procedure. Following intubation he was mechanically ventilated in ICU until the tracheostomy was 
performed. He received intravenous midazolam $2-4 \mathrm{mg} /$ hour initially in ICU as he was not tolerating the endotracheal tube and was kept on spontaneous mode with a $\mathrm{FiO}_{2}$ 0.3, pressure support of $8 \mathrm{cmH}_{2} \mathrm{O}$ and a PEEP of $5 \mathrm{cmH}_{2} 0$. His respiratory rate varied between $14-18$ breaths per minute. His arterial oxygen saturation was between 96-99\%. Within 24 hours of admission to the ICU a tracheostomy was performed in the operating theatre by the surgical team under general anaesthesia.

Following recovery from anaesthesia he was kept breathing spontaneously on a tracheostomy mask with $\mathrm{FiO}_{2}$ 0.28. He maintained oxygen saturation between 95-99\%. Human tetanus immune globulins 5000 units along with intravenous metronidazole $500 \mathrm{mg}$ was administered 8 hourly for 10 days.

In less than 24 hours of admission to ICU he developed painful muscle spasms with episodes of desaturation which indicated that the disease was progressing to grade II (moderate severity). $\mathrm{He}$ therefore required ventilator support and he received synchronised intermittent mandatory ventilation (SIMV) on the same ventilator settings prior to tracheostomy but with a $\mathrm{FiO}_{2}$ of 0.4. Arterial blood gas values remained within normal range. We continued to ventilate him alternatively on SIMV and spontaneous modes $\left(\mathrm{FiO}_{2} \mathrm{O} .4\right.$, pressure support $8 \mathrm{cmH}_{2} \mathrm{O}$ and PEEP $5 \mathrm{cmH}_{2} \mathrm{O}$ ) for 2 days and weaned him on to a tracheostomy mask by the 3rd day in ICU.

\section{Magnesium therapy}

With the development of spasms on day 1 we commenced magnesium therapy with careful monitoring of urine output and patellar reflex. A loading dose of intravenous magnesium sulphate $5 \mathrm{~g}$ over 30 minutes was followed by $2 \mathrm{~g} /$ hour infusion. Serum magnesium levels were monitored 6 hours after commencing the magnesium infusion. However on day 5 of ICU stay the rate of infusion had to be increased by $0.5 \mathrm{~g} 8$ hourly up to a maximum of $3 \mathrm{~g} /$ hour to control his intractable spasms and rigidity and once controlled we reduced the infusion rate to $2 \mathrm{~g} /$ hour. We were successful in controlling his spasms during the next 2 weeks purely with magnesium sulphate titrated to effect. No sedatives or opiates were given. He remained conscious throughout this period and would respond to command which enabled optimum nursing care.

Serum magnesium levels were measured twice or thrice daily initially, but due to limited resources it could not be done on certain days, especially towards the latter stages of his ICU stay. It was reassuring to note that despite high serum magnesium levels which was evident in this patient from the beginning (Figure 1), clinically his respiratory rate \& tendon reflexes were not diminished except on two occasions on day 4 (serum $\mathrm{Mg} 5.5 \mathrm{mg} / \mathrm{dl}$ ) and day 5 (serum $\mathrm{Mg}$ $5.8 \mathrm{mg} / \mathrm{dl}$ ) of ICU stay which compelled us to withhold the magnesium infusion for 2-3 hours. Serum magnesium levels varied between 5.2 and $6.8 \mathrm{mg} / \mathrm{dl}$ in the first 12 days and reached $7.4 \mathrm{mg} / \mathrm{dl}$ between days 13 to 17 .

From the $3^{\text {rd }}$ day onwards he was breathing spontaneously via a tracheostomy mask with supplementary oxygen $(0.28)$.

On the $5^{\text {th }}$ day in ICU he developed episodes of labile blood pressure and fluctuating heart rates indicating autonomic instability. According to the Ablett classification he now belonged to grade III (severe) disease severity. 3 bolus doses of atropine $0.6 \mathrm{mg}$ were administered during 24 hours to counteract the low heart rate. However he did not require vasopressors and no antihypertensive medication were required. Magnesium sulphate infusion was increased up to $2.5 \mathrm{~g} /$ hour during periods of high blood pressure with close and continuous monitoring of respiratory rate, tendon reflexes and hourly urine output. Magnesium infusion was withheld for approximately 2 hours on day 5 as the patellar reflex could not be elicited.

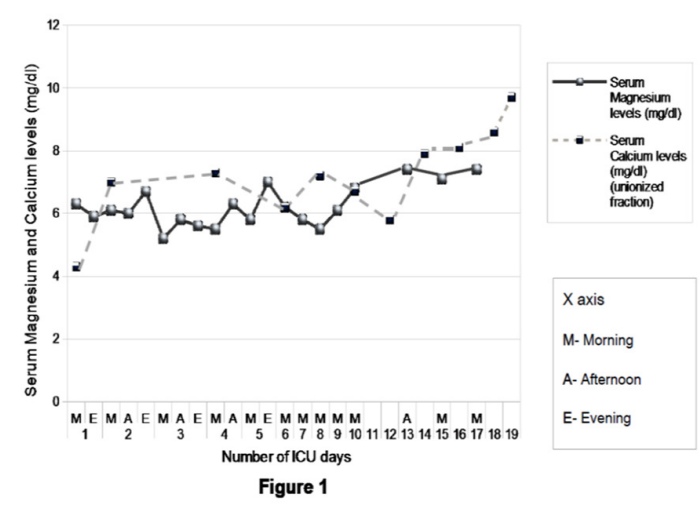

*Normal serum magnesium Level $=1.8-3.0 \mathrm{mg} / \mathrm{dl}$

$*$ Normal serum calcium Level $=8.6-10.7 \mathrm{mg} / \mathrm{dl}$ 
Serum calcium levels were monitored once in 3 days while the patient was on the magnesium infusion. It ranged between $4.3 \mathrm{mg} / \mathrm{dl}$ on day 1 and $9.7 \mathrm{mg} / \mathrm{dl}$ on day 19 . Intravenous calcium chloride $10 \% 10 \mathrm{ml}$ was administered over a 10 minute period on day 1,6,10 and 12. Despite low levels of serum calcium during his ICU stay clinically there were no signs suggestive of hypocalcaemia.

\section{Intensive care management}

Benzodiazepines were not used during his ICU stay except initially when he was electively intubated prior to performing the tracheostomy. Bolus doses of intravenous fentanyl 25 micrograms were used prior to suctioning of the tracheostomy tube, initially at a frequency of 5-6 times per day. Rest of the ICU care bundles were implemented with special emphasis on nutrition which was initiated from day 1 in ICU and the use of thromboembolic deterrent stocking (TEDs) and subcutaneous enoxaparin 40mg daily at night. Continuous monitoring (Figure 2 and 3) and relevant investigations were done on a daily basis.
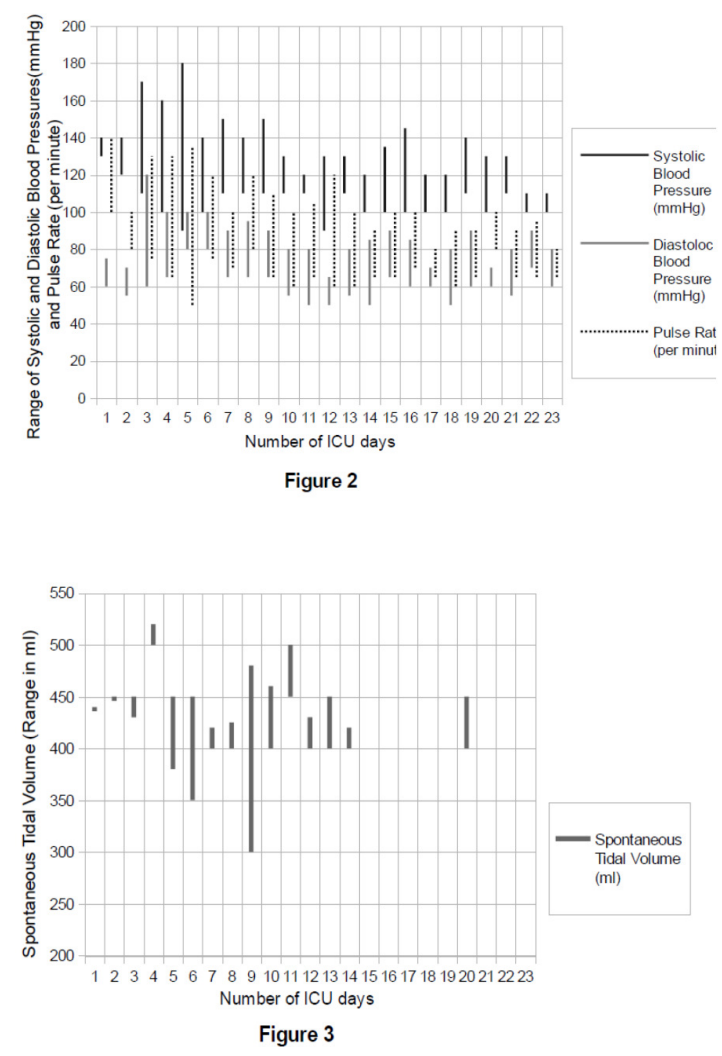

His haemoglobin levels ranged from $11.1 \mathrm{~d} / \mathrm{dl}$ on day 1 to $7.8 \mathrm{~g} / \mathrm{dl}$ on day 20 , the lowest value noted during his ICU stay, for which he was prescribed haematinics.

Platelet counts were within normal limits until day 12 and thereafter the trend was on the rise from $613,00010^{9}$ per litre on day 13 to 1143,000 $10^{9}$ per litre on day 19 which was suggestive of reactive thrombocytosis. He was prescribed a daily dose of aspirin $75 \mathrm{mg}$ and the count prior to discharge from ICU was $756,00010^{9}$ per litre. The blood picture confirmed the diagnosis along with normocytic normochromic anaemia and roulex formation and white blood cells showing an absolute neutrophil leucocytosis with platelet anisocytosis.

\section{Complications}

He developed fever on day 7 for which intravenous co-amoxyclavulanic acid $1.2 \mathrm{~g} 8$ hourly was commenced empirically. The white cell count was within normal range until day 10 and on day 11 his count increased to $16.2310^{9}$ per litre associated with fever and a CRP of $96 \mathrm{mg} / \mathrm{dl}$. Blood and urine were negative for isolates. Tracheal secretion cultures sent on day 11 were positive for coliforms and co-amoxyclavulanic acid was replaced by intravenous meropenam $500 \mathrm{mg} 8$ hourly (as per the sensitivity results) for 7 days to which he responded.

After a period of 11 days in ICU he became agitated (pulling out the intravenous cannula and catheter) restless and delirious despite normal metabolic and haemodynamic parameters. Intravenous haloperidol $2.5 \mathrm{mg} 8$ hourly (PRN) was prescribed for 48 hours which helped him to overcome this abnormal behavioural pattern.

\section{Recovery}

By 2 weeks oral feeds were well established and although his rigidity persisted, we were able to mobilise him out of bed. He gradually started to walk, initially with the help of the physiotherapist and ICU staff.

With cessation of spasms, magnesium sulphate infusion was titrated to effect and subsequently tailed off and omitted on day 19.

He was successfully discharged to the surgical ward at the end of 3 weeks after de-cannulation of the tracheostomy and supportive treatment. 


\section{Discussion}

A role for magnesium in the management of tetanus has been postulated by many authors. ${ }^{1,6,7}$ On the other hand some advocate sedation with benzodiazepines for the control of spasms with the use of muscle relaxants when necessary., ${ }^{2,3}$ Often magnesium is used mainly as an adjunct to reduce the requirement of other drugs ${ }^{7}$ and for the control of sympathetic over-activity when alpha and beta blockers are inadequate. Many reviewers ${ }^{3,8}$ stress that magnesium is inadequate used alone and does not reduce mortality. This was supported by a randomised double blind placebo controlled trial which revealed that magnesium infusion does not reduce the need for mechanical ventilation in adults with severe tetanus but does reduce the requirement for other drugs to control muscle spasms and cardiovascular instability. ${ }^{7}$ Thus magnesium used as the sole agent both for the control of spasms and sympathetic over-activity is still not standard practice.

We followed the protocol recommended by Attygalle and Rodrigo where magnesium is used as first line and mainstay therapy for both the control of spasms and sympathetic overactivity. ${ }^{6}$

Studies have shown that magnesium therapy has to be continued for few days after the cessation of spasms as attempts at withdrawal resulted in unacceptable rigidity in some patients and sustained tachycardia in others. ${ }^{1}$ We continued magnesium therapy until day 19 even though he was free of spasms after two weeks of therapy. He did however exhibit mild rigidity even at the time of discharge to the ward but this did not interfere with his daily activities.

Magnesium as the sole therapy meets many of the requirements where conventional treatment fails. ${ }^{9}$ The fact that magnesium can control spasms and sympathetic over activity while patients remain conscious and cooperative and mobilised enable better nursing care which contributes to a better outcome avoiding the dreaded complications of over-sedation and this was apparent in our patient.

We realised that even though the serum magnesium levels were above the therapeutic range clinical judgement showed no ill effects. Rarely did we have to withhold magnesium therapy despite high serum concentrations of magnesium as the tendon reflexes which were monitored hourly at the beginning were intact in most instances and there was no evidence of respiratory insufficiency while on magnesium therapy. Since the patient remained conscious and was not paralysed the dose of magnesium could be titrated according to the clinical signs rather than using serial levels of magnesium.

In a resource limited setting the management of tetanus is challenging. A lesson learnt was that if we closely monitor patient's patellar reflexes, respiratory rate while breathing spontaneously and hourly urine output we may not need to depend on serum magnesium levels when managing such patients.

Our patient was a farmer with an incomplete immunization record and immunity to tetanus by the combined vaccine may not be life long and booster injections should be given after individuals sustain tetanus prone wounds.

Tetanus is a rare disease today and randomised controlled trials to determine the benefit of one treatment regime over another are not easily performed. Therefore case reports such as this would be useful in adding to the accumulating evidence of the efficacy and safety of magnesium as first line and mainstay therapy. The avoidance of benzodiazepines to achieve an awake patient in ICU is of tremendous advantage and also using magnesium as the sole agent, which is relatively inexpensive in comparison to benzodiazepines in the management of such patients is cost effective especially in a resource limited setting.

\section{Acknowledgements}

I thank Dr. Deepthi Attygalle for the advice given during the management of this patient and to Dr. Nalini Rodrigo for her assistance in editing and involvement in analysing this case report critically for important intellectual content.

I am also grateful to Dr.K.M.K.C Bandara for his contribution in designing the graphic material.

\section{References}

1. Attygalle D, Rodrigo N. Magnesium as first line therapy in the management of tetanus: a prospective study of 40 patients. Anaesthesia. 2002;57:778-817

http://dx.doi.org/10.1046/j.1365-

2044.2002.02698_6.x 
2. Taylor A M, Tetanus, Continuing Education in Anaesthesia, Critical Care and Pain. 2006;3:1014

3. Cook TM, Protheroe RT, Handel JM. Tetanus: a review of the literature. $\mathrm{Br} \mathrm{J}$ Anaesthesia. 2001;87:477-87

http://dx.doi.org/10.1093/bja/87.3.477

4. Ratnayaka RMKN, Ranaweera J, Palihakkara P, Ranathunga PEAN. Case report on profound central depression during treatment of tetanus with combined magnesium, midazolam, morphine \& fentanyl. Sri Lanka Journal of Anaesthesiology. 2015;23:86-88 http://dx.doi.org/10.4038/slja.v23i2.8077

5. Mathew P J, Samrawig J. Magnesium sulphate for treatment of tetanus in adults. Anaesth Intensive Care. 2010 Jan;38(1):185-9

PMid:20191795

6. Attygalle D, Rodrigo N. Magnesium sulphate for control of spasms in severe tetanus. Can we avoid sedation \& artificial ventilation? Anaesthesia. 1997;52:956-62

http://dx.doi.org/10.1111/j.1365-2044.1997.206az0340.x

PMid:9370837

7. Thwaites CL, Yen LM, Lean HT, et al. Lancet. 2006;368: 1436-43 http://dx.doi.org/10.1016/S0140-6736(06)69444-0

8. Attygalle D, Rodrigo N. Review- New trends in the management of tetanus, www.futuredrugs.com

9. Attygalle D, Rodrigo N. The value of a conscious patient in the intensive care management of tetanus-maximizing magnesium \& minimizing midazolam. Sri Lanka Journal of Anaesthesia. 2015 July;23(2):50-55

http://dx.doi.org/10.4038/slja.v23i2.8089

(C) 2016. Dassanayake. This is an Open Access article distributed under the terms of the Creative Commons Attribution License (http: //creativecommons.org/licenses/by/4. 0), which permits unrestricted use, distribution, and reproduction in any medium, provided the original work is 